\section{$\underset{\substack{\text { hommes } \\ \text { \& migrations }}}{ }$}

\section{Hommes \& migrations}

Revue française de référence sur les dynamiques

migratoires

$1320 \mid 2018$

Au prisme de la consommation

\title{
Les migrations internationales et leurs effets
}

\section{Amal Miftah}

\section{(2) OpenEdition \\ Journals}

\section{Édition électronique}

URL : https://journals.openedition.org/hommesmigrations/4067

DOI : 10.4000/hommesmigrations.4067

ISSN : 2262-3353

\section{Éditeur}

Musée national de l'histoire de l'immigration

\section{Édition imprimée}

Date de publication : 1 janvier 2018

Pagination : 114-120

ISBN : 978-2-919040-40-7

ISSN : $1142-852 X$

\section{Référence électronique}

Amal Miftah, «Les migrations internationales et leurs effets », Hommes \& migrations [En ligne], 1320 | 2018, mis en ligne le 01 janvier 2018, consulté le 28 juin 2022. URL : http://journals.openedition.org/ hommesmigrations/4067; DOI : https://doi.org/10.4000/hommesmigrations.4067 


\section{LES CHANTIERS DE LA RECHERCHE}

\section{LES MIGRATIONS INTERNATIONALES ET LEURS EFFETS}

Par AMAL MIFTAH, chercheure associée, université Paris Dauphine, LEDa, DIAL UMR 225.

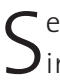
elon les Nations unies, le nombre de migrants internationaux - c'est-à-dire de personnes vivant dans un pays autre que celui où elles sont nées - a atteint 244 millions en 2015, soit une augmentation de $41 \%$ par rapport à 2000 . Bien qu'une grande partie de ces migrants habitent dans un pays du Nord, ceux originaires du Sud et habitant dans le Nord ne représentent qu'un peu plus d'un tiers de l'ensemble des migrants internationaux' Il apparaît, en effet, que dans de nombreuses parties du monde la migration s'effectue souvent entre pays de la même zone géographique (en Afrique par exemple). Parmi les grandes tendances migratoires, on peut citer en particulier la féminisation croissante des migrations et la forte émigration des travailleurs les plus qualifiés. Dernièrement, le nombre de femmes et de travailleurs qualifiés a enregistré une forte augmentation, même si leur proportion reste plus élevée au Nord qu'au Sud. L'image du migrant comme homme, jeune, actif, célibataire et sans qualification ne semble donc plus correspondre à la réalité.

Au sein des pays de l'Organisation de coopération et de développement économiques (OCDE), le pourcentage d'immigrés passés par l'enseignement supérieur était, en 2010-2011, d'environ $30 \%$, soit une augmentation de deux tiers en dix ans. Le continent africain reste très marqué par cette tendance migratoire : $13 \%$ des migrants d'origine sub- saharienne et diplômés du supérieur vivent dans les pays de l'OCDE. On observe ainsi une sorte de « sélection » de la migration par rapport au niveau d'éducation. Cette situation amène à considérer le risque de la "fuite des cerveaux ». Étant donnée l'importance des taux d'émigration des plus qualifiés, dépassant les taux d'émigration totaux pour presque tous les pays d'origine (à l'exception des immigrés mexicains par exemple), il est plausible de penser que l'exode des cerveaux est un phénomène toujours en perpétuelle expansion. Ceci est particulièrement inquiétant, d'autant plus que les pays d'origine ont lourdement investi dans l'enseignement supérieur au cours de la décennie écoulée. C'était le cas de nombreux pays du MoyenOrient et d'Afrique du Nord. Cette situation représente un défi et un risque pour plusieurs pays d'origine, d'autant que les personnes qualifiées sont devenues récemment la cible privilégiée des politiques d' «immigration choisie » des pays d'accueil. En fait, des changements substantiels se sont produits en matière de politique migratoire depuis les années 1980. Ainsi, plusieurs pays de destination ont adopté une politique d'immigration sélective fondée sur des critères de capital humain. C'est le cas, par exemple, de l'Australie, du Canada et, dans une moindre mesure, du Royaume-Uni. En même temps, face à la pression migratoire, plusieurs pays d'accueil ont

1. En 2013, 136 millions de migrants internationaux résidaient dans l'hémisphère Nord et 96 millions dans l'hémisphère Sud: 82,3 millions ont effectué des migrations Sud-Sud, tandis que 81,9 millions auraient migré du Sud vers le Nord. Voir Nations Unies,

"Tendances des migrations internationales", 2013 
tenté de contrôler l'immigration et indirectement se sont peu à peu ouverts à la problématique de développement des pays du Sud.

Enfin, notons qu'au cours des dernières décennies, en raison de la situation sociale et économique explosive, et de l'augmentation des flux migratoires et des envois de fonds des migrants, les questions liées aux migrations ont fait l'objet d'une attention accrue de la part des gouvernements de plusieurs pays d'origine. Un certain nombre de ces pays ont privilégié, en accord avec les pays d'accueil, l'emploi temporaire à l'étranger et le retour définitif des migrants. C'est le cas des Philippines, par exemple, qui, en signant des accords avec de nombreux pays de destination, fournissent des contrats de travail de courte durée aux Philippins qui n'ont pas le droit, en contrepartie, de se faire rejoindre par le reste de leur famille. L'exemple chinois ou indien est aussi très intéressant. À partir des années 1980, on a observé, grâce aux politiques d'aide du gouvernement chinois, une vague d'émigration chinoise qui concernait les étudiants, les travailleurs hautement qualifiés et les professionnels.

\section{Revue de la littérature sur les effets des migrations sur les pays d'origine}

La question des effets de la migration sur les pays d'origine des migrants a largement été étudiée dans la littérature. Dans les années 1960, les premières réflexions de la littérature économique sur ce sujet ont reposé sur le modèle de l'économie néoclassique. Le cadre théorique est structuré autour du schéma de développement initié par l'économiste américain Walt W. Rostow dans son ouvrage Les étapes de la croissance économique (1960). Rostow a insisté sur le caractère évolutif du mouvement d'une société traditionnelle vers une société moderne. La convergence économique des pays et les transformations structurelles peuvent se faire grâce à la mobilité des personnes et des capitaux. Ces flux migratoires sont généralement originaires des pays moins développés, où le facteur travail est abondant et le facteur capital plus rare ; la mobilité d facteur travail conduit à un équilibre entre l'offre et la demande de la maind'œuvre. Ces théories ont donc tendance à minimiser l'importance des effets négatifs des migrations. L'analyse du rôle des migrations dans le développement des pays d'origine a connu un revirement important quelques années après. En fait, un courant pessimiste a prévalu durant de nombreuses années voire des décennies. Les opinions pessimistes ont été inspirées par la théorie de la dépendance, initiée par Jagdish Bhagwati dans les années 1970. Elles soutiennent que le système mondial se caractérise par des mécanismes d'exploitation et de pillage des pays sous-développés par les pays développés, manifestés par une récupération des ressources naturelles et humaines de la périphérie par le centre, ce qui aggrave les problèmes de sous-développement. Ces théories vont se développer parallèlement aux réflexions sur le «brain drain » ou la fuite des cerveaux. Sur la même lancée, les théories de la croissance endogène, émergent dans les années 1980 et même 1990, suggèrent que la migration est un phénomène ralentissant la croissance économique des pays en développement. Plus précisément, elles considèrent que l'émigration joue défavorablement sur la productivité et la richesse des pays d'origine car elle cause une perte globale de capital humain à ces pays. Il est important de noter que, jusqu'aux années 1990, les données harmonisées sur les migrations internationales par niveau d'éducation ou de qualification étaient absentes. Par conséquent, ces modèles ont été plus descriptifs qu'analytiques et semblent insuffisants pour analyser l'incidence de la fuite des cerveaux.

Dans les années 1980 et 1990, avec la hausse ininterrompue des montants des envois de fonds des migrants - dépassant même, dans certains cas, l'aide publique au développement et les investissements directs étrangers -, des travaux empiriques de plus en plus nombreux ont montré l'impact positif de la migration sur le 


\section{LES CHANTIERS DE LA RECHERCHE}

développement des pays d'origine si les conditions économiques et politiques y sont favorables. Ces migrants qui transfèrent de l'argent sont ainsi perçus comme des acteurs du développement; leurs transferts sont parfois au cœur des dynamiques de développement local. Dans le sud du Maroc par exemple, les villageois ont créé des associations de développement par le biais desquelles ils sollicitent les migrants installés en France pour le montage financier et technique des projets. Cette mise en commun de ressources financières et techniques a permis l'électrification des foyers de la vallée du Massa et la création d'un système d'alimentation des maisons en eau potable ${ }^{2}$.

Aujourd'hui encore, l'impact de la migration sur le pays de départ ou sur celui d'accueil reste controversé et difficile à évaluer, car les migrations ont des effets parfois indirects (spillovers) et échelonnés dans le temps. De plus, les gains dépendent en grande partie de la nature du flux migratoire : migration temporaire versus permanente, migration hautement qualifiée ou regroupement familiale.

Les migrations temporaires sont perçues par la plupart des pays d'origine comme étant bénéfiques pour promouvoir leur développement. Cette contribution des migrants à l'économie nationale est due certes aux transferts de fonds, mais aussi aux transferts de compétence et de connaissances, à la création d'entreprises et aux réseaux diasporiques. Au-delà de ces effets, les migrations peuvent amener à la diffusion de normes et de valeurs sociales (valeurs démocratiques par exemple) dans les pays d'origine, d'où l'intérêt d'étudier tous les effets potentiels des migrations. Aussi, il conviendrait d'étudier et de comprendre à la fois les causes et les conséquences des migrations.

\section{Les transferts de fonds}

Selon les données de la banque mondiale, les envois de fonds officiellement enregistrés vers les pays en développement se sont élevés à 431,6 milliards de dollars en 2015. Exprimés en pourcentage du produit intérieur brut (PIB), ces fonds sont particulièrement importants pour des petits pays comme le Cap-Vert ou Haïti. Dans certains cas, ils présentent l'avantage pour les pays qui les reçoivent d'être plus stables que les autres sources de financement extérieur et d'offrir un soutien à la consommation des ménages, surtout pendant les périodes difficiles. De manière générale, les études empiriques concluent qu'au niveau microéconomique, les transferts des migrants permettent essentiellement de satisfaire les besoins de base de la famille et d'améliorer son bien-être économique et social3. Dans plusieurs cas, ces ressources permettent à la famille restante de faire face aux éventuels chocs socioéconomiques négatifs auxquels elle est exposée (maladie, chômage, mauvaise récolte, etc.) et d'entreprendre de nouveaux projets. En effet, selon la Nouvelle économie de la migration du travail (NEMT), la migration est une décision collective prise au niveau du ménage, elle vise la maximisation des revenus et la minimisation des risques encourus par la communauté d'origine en l'absence ou avec la défaillance des marchés financier, d'assurance et du travail, en particulier grâce aux transferts de fonds 4 . À cet égard, les transferts des migrants semblent contribuer à la réduction de la pauvreté transitoire des ménages à faible revenu et au développement de leur capital humain à travers

\footnotetext{
2. Thomas Lacroix, "Le développement au carrefour des exils marocains ", in Christophe Jaffrelot, Christian Lequesne (dir.), L'enjeu mondial. Les migrations, Paris, Presses de Sciences Po, 2009. 3. Il est important de noter que, pour examiner les conséquences des transferts des migrants sur les pays d'origine, il est essentiel de garder à l'esprit les raisons qui poussent ces individus à envoyer des fonds à leur famille restée au pays ainsi qu'à leur communauté d'origine. Voir, par exemple : Hillel Rapoport, Frédéric Docquier, "The economics of migrants' remittances", in Serge-Christophe Kolm, Jean Mercier Ythier (dir.), Handbook of the Economics of Giving, Altruism and Reciprocity, Amsterdam, North Holland, 2006, pp. 1135-1198; Jamal Bouoiyour, Amal Miftah, "Why do migrants remit? Testing hypotheses for the case of Morocco", in IZA Journal of Migration, vol. 4, n², 2015. 4. J. Edward Taylor, "Migrations: nouvelles dimensions et caractéristiques. Causes, conséquences et répercussions en termes de pauvreté rurale ", in Kostas G. Stamoulis (dir.), Alimentation, agriculture et développement rural. Problèmes actuels et émergents en matière d'analyse économique et de recherche de politiques ", Rome, FAO, 2001.
} 
notamment l'amélioration de leur accès à la santé et à l'éducation's.

Cependant, les résultats des études sont souvent contradictoires. Elles introduisent d'ailleurs une condition nécessaire pour que les envois de fonds puissent réduire la pauvreté : les migrants doivent provenir de régions pauvres où le nombre de migrants est élevé. En évaluant les effets des variations marginales des transferts de fonds sur la pauvreté dans des régions mexicaines ayant des niveaux de migration différents, Taylor et al. ${ }^{6}$ arrivent à la conclusion que, dans les régions rurales, l'élargissement de l'accès à la migration a pour conséquence un effet beaucoup plus bénéfique des transferts des migrants sur la répartition des richesses. Le développement des réseaux d'émigration semble faciliter l'émigration des pauvres et contribuer, par conséquent, à la réduction des inégalités. La NEMT a également mis en lumière la nécessité de considérer les différents impacts souvent indirects de la migration sur la communauté entière. Comme l'indique Taylor, il existe dans le cas du Mexique " un effet d'entraînement des dépenses qui favorise la transmission de nombreux bénéfices dérivés des envois de fonds à des familles autres que les destinataires, au sein et hors de l'économie rurale. Les ménages ruraux pauvres sont au nombre des bénéficiaires de ces envois7 ". En d'autres termes, il est nécessaire de prendre en compte les effets multiplicateurs indirects de la migration et des envois de fonds sur les communautés d'origine dans leur ensemble.

De nombreuses analyses des impacts de la migration se limitent en fait à l'utilisation des transferts par les ménages destinataires et à leur impact direct dans les zones de départ, et elles concernent souvent les pays d'Amérique latine. Toutefois, il existe relativement peu d'études convaincantes portant sur l'effet de ces revenus sur l'éducation ou le travail des enfants par exemple. II ressort néanmoins de ces études que les ménages qui reçoivent des transferts de l'étranger sont plus enclins à investir dans l'éducation de leurs enfants et moins enclins à les faire travailler. Mais, une distinction doit être faite entre les effets des envois de fonds et ceux liés à la migration internationale. À titre d'exemple, l'impact des migrations sur l'éducation des enfants peut s'avérer négatif car la migration des adultes pourrait augmenter la responsabilité des enfants les plus âgés (surtout les garçons) et les obliger à travailler et à réduire le temps investi dans l'éducation (en particulier dans les zones rurales). On peut imaginer que, dans une telle configuration, l'investissement dans l'éducation a, en plus du coût de financement habituel (frais de scolarité, manuels scolaires, etc.), un coût d'opportunité pour les parents, celui des salaires des enfants auxquels ils renoncent.

Au niveau macroéconomique, l'évaluation des effets des migrations sur le pays d'origine est plus difficile et les résultats empiriques sont mitigés. Certaines études montrent que les envois de fonds peuvent jouer un rôle positif sur la croissance économique, la répartition des revenus et la réduction des déficits de la balance commerciale ${ }^{8}$. D'autres études estiment que les transferts des migrants peuvent avoir des effets négatifs sur les sociétés d'origine. Dans cet ordre d'idées, certains auteurs insistent sur la baisse de la productivité de ceux qui les reçoivent, en

5. Ghazala Mansuri, "Migration, school attainment and child labor: evidence from rural Pakistan ", in Policy Research Working Paper Series, $n^{\circ}$ 3945, Washington DC, The World Bank, 2006 ; Barry McCormick, Jackline Wahba, "Overseas work experience, savings and entrepreneurship amongst return migrants to LDCs", in Journal of African Economies, vol. 12, n 4, 2003, pp. $500-532$; Jamal Bouoiyour, Amal Miftah, "Migration, remittances and educational levels of household members left behind: Evidence from rural Morocco ", in European Journal of Comparative Economics, vol. 12, $n^{\circ}$ 1, 2015, pp. 21-40. 6. J. Edward Taylor, Jorge Mora, Richard Adams, Alejandro Lopez-Feldman, "Remittances, inequality and poverty: Evidence from rural Mexico ", in Working Papers, $n^{\circ}$ o5-003, University of California, Department of Agricultural and Resource Economics, 2005. 7. J. Edward Taylor, op. cit. 8. Bachika Fayissa, Christian Nsiah, "Can remittances spur economic growth and development? Evidence from Latin American Countries ", Department of Economics and Finance Working Paper Series, Middle Tennessee State University, 2010 ; Dilip Ratha, « Enhancing the developmental effect of workers'remittances to developing countries », in Global Development Finance, Washington DC, The World Bank, 2004 ; Edgard R. Rodriguez, "International migrants' remittances in the Philippines ", in Canadian Journal of Economics, vol. 29, Special Issue : Part 2, 1996, pp. 427-432. 


\section{LES CHANTIERS DE LA RECHERCHE}

plus de l'apparition d'une certaine dépendance vis-à-vis de ces fonds9. D'autres chercheurs pensent que les transferts peuvent favoriser la croissance de la demande domestique, qui stimulera, à son tour, les importations au détriment de la production locale. Une entrée massive de devises étrangères pourrait aussi provoquer l'appréciation de taux de change réel et la baisse de la compétitivité de l'économie ${ }^{10}$ Ce phénomène porte le nom du syndrome hollandais.

\section{Migrations de retour et migrants qualifiés}

Les migrants qui reviennent dans leurs pays ainsi que les migrants qualifiés, de par leur capital social, humain, scientifique et économique, peuvent, si certaines conditions sont réunies, contribuer au développement de leur pays d'origine. Ceci s'explique en grande partie par leurs connaissances des opportunités d'investissement existant dans les pays d'origine, grâce surtout, à leurs réseaux et aux liens qu'ils entretiennent avec leur société d’origine. En effet, la diaspora la plus éduquée peut contribuer à renforcer les échanges commerciaux, intellectuels et culturels entre le pays de départ et le reste du monde. Aujourd'hui, les schémas de mobilité internationale se transforment du fait de l'amélioration et de la réduction des coûts de transport et de l'information, et de l'apparition de nouveaux moyens de communication. Plus mobiles, plus connectés et souvent plus compétents, les migrants peuvent agir en faveur du développement de leur pays d'origine. Les réseaux diasporiques deviennent donc de plus en plus des acteurs importants du développement des communautés d'origine en finançant des projets et en soutenant les initiatives locales.

Comme nous l'avons déjà souligné, la littérature économique portant sur les conséquences de l'émigration des migrants qualifiés a longtemps insisté sur les effets négatifs de l'exode des cerveaux. Cette émigration était perçue par certains comme un pillage de la main-d'œuvre qualifiée des pays les moins développés. Très récemment, quelques études empiriques ont montré que la migration qualifiée n'est pas qu'un fléau, elle peut être accompagnée d'un brain gain (gain de cerveaux). Beine et al." mettent en évidence les effets favorables de l'émigration qualifiée sur 50 pays en développement. Les auteurs confirment l'existence d'un effet d'incitation à la formation du capital humain dans ces pays. Autrement dit, les perspectives de migration, en augmentant le rendement espéré du capital humain, peuvent accroître l'investissement dans l'éducation et les incitations à acquérir des qualifications au Sud. Ce type d'émigration a aussi un effet positif sur la croissance des pays caractérisés par un faible taux d'émigration de qualifiés (inférieur à $20 \%$ ) et/ou par une proportion des diplômés de l'enseignement supérieur inférieure à $5 \%$. Cependant, un effet négatif serait davantage susceptible de se matérialiser en cas de migration d'une part non négligeable de la population ou si le rendement de l'éducation dans le pays d'accueil était faible. Toujours dans le même esprit, Batista et al. ${ }^{12}$ testent l'effet de la probabilité de migrer sur le niveau scolaire atteint, en utilisant des données d'enquête collectées sur les migrants originaires du Cap-Vert. Leurs résultats montrent bien que la probabilité de migrer augmente la probabilité d'atteindre le niveau d'enseignement secondaire. 
En matière de migration de retour, la littérature empirique existante suggère qu'elle peut avoir des effets positifs sur les membres de la famille et la communauté d'origine des migrants. Il semble, par exemple, que le retour des migrants a augmenté les investissements dans certains pays en développement comme l'Égypte ${ }^{13}$ et la Tunisie ${ }^{14}$. Pour certains auteurs comme Lucas et Stark'5 ou Poirine ${ }^{16}$, le fait de vouloir retourner dans son pays a pour effet une augmentation des envois de fonds orientés vers l'investissement en capitaux fixes, comme l'achat d'une maison, de terres ou de bétail, et en capitaux dits publics, comme les relations avec la famille et la communauté d'appartenance. De même, l'existence d'un héritage garantira le maintien des transferts de fonds sur le long terme. Dans le cas des migrants originaires de la République dominicaine, De la Brière et al. ${ }^{17}$ constatent que le migrant qui désire y retourner investit plus dans le but d'hériter du patrimoine de la famille.

\section{Migration internationale et transfert de normes culturelles et sociopolitiques}

Au-delà de ses effets "économiques ", la migration internationale peut jouer un rôle non négligeable dans les changements sociopolitiques et culturels dans les pays d'origine. Certaines études sur le Maroc, par exemple, montrent l'importance de la migration dans l'acquisition de capital humain ${ }^{18}$. II semble aussi que le migrant ait contribué aux changements dans les habitudes de consommation et dans les comportements des individus ${ }^{19}$, laissant présager un processus de transfert de normes. On sait que les normes se construisent par leur diffusion entre les agents par l'intermédiaire des réseaux de diffusion et que les réseaux transnationaux sont eux aussi susceptibles de jouer un rôle dans la diffusion et la socialisation de la norme.

Quelques recherches très récentes ont prêté attention aux effets non économiques de la migration internationale sur les pays d'où partent les migrants. Ces effets couvrent diverses dimensions telles que la qualité des institutions ${ }^{20}$, la fertilité ${ }^{21}$ et la démocratie ${ }^{22}$. Le travail de Spilimbergo ${ }^{23}$ fournit une contribution majeure à la littérature existante en démontrant le rôle joué par les étudiants qui ont choisi de quitter leur pays natal pour aller étudier à l'étranger, une minorité très influente, dans l'évolution des régimes politiques de leur pays d'origine.

\section{Conséquences économiques de l'immigration : le cas de la France}

Au-delà des enjeux soulignés plus haut, les conséquences des migrations peuvent aussi être nombreuses pour les pays d'accueil. En Europe, face au vieillissement de la population active, plusieurs économistes se sont interrogés sur la nécessité d'une « migration de remplacement » pour pallier cette population manquante. En réalité, de nombreux pays européens sont confrontés à la réduction des taux de natalité et à l'allongement de 


\section{LES CHANTIERS DE LA RECHERCHE}

l'espérance de vie. La France reste cependant, avec I'Irlande, le pays le plus fécond d'Europe, même si elle connaît, elle aussi, une forte augmentation de la part des 65 ans et plus dans la population. ’̀ cause de cette situation démographique inquiétante, l'équilibre financier des systèmes de retraite par répartition en Europe pourrait être difficile à atteindre. Afin de contrecarrer ce processus du vieillissement des populations, les débats s'articulent souvent autour du rôle de la migration des jeunes dans la croissance de la population active. Dans son rapport annuel sur les Perspectives des migrations internationales de 2012, l'OCDE explique qu'entre 2000 et 2010 , la croissance de la population active dans certains pays (Suisse, Italie, Luxembourg et Royaume-Uni) résulte intégralement ou presque de l'arrivée de nouveaux immigrés.

Contrairement à la France, un grand nombre de travaux sur les conséquences économiques de l'immigration porte sur les États-Unis, où le sujet a été investi depuis maintenant plusieurs décennies ${ }^{24}$. L'immigration en France est souvent accusée d'être responsable du chômage mais la réalité des flux migratoires est trop souvent méconnue. Il est vrai que la migration pour motif économique a progressé ces dernières années mais elle reste minoritaire, ne dépassant pas $10 \%$ de l'ensemble des admissions au séjour accordées aux ressortissants de pays tiers, alors que plus de la moitié du flux d'entrées s'explique par des motifs familiaux. Cette immigration familiale, qui « s'impose » à la
France en vertu du droit fondamental de vivre en famille ne peut pas être gérée en lien avec les objectifs économiques. Des économistes ont alors essayé d'estimer l'impact de l'immigration sur le marché du travail français. Les résultats varient substantiellement d'une étude à l'autre. Dans son étude sur les conséquences du rapatriement des pieds noirs d'Algérie en 1962 , Hunt ${ }^{25}$ compare l'évolution des taux de chômage et de salaires dans les départements français et souligne qu'une augmentation du nombre de rapatriés aurait augmenté le chômage des natifs de 0,2 \% mais aussi le salaire annuel moyen de $1,3 \%$. D'autres auteurs comme Ortega et Verdugo ${ }^{26}$ constatent un effet beaucoup plus positif de l'immigration. Ainsi, ayant étudié la période allant de 1962 à 1999, les auteurs soulignent qu'une croissance de $10 \%$ de l'immigration augmente le salaire des natifs de $3 \%$. Selon les auteurs, l'immigration entraîne aussi une réallocation des natifs vers des emplois de meilleure qualité.

En France, l'immigration est aussi très souvent perçue comme un élément aggravant le déficit public. L'étude de Chojnicki et Ragota ${ }^{27}$ a tenté d'évaluer la contribution de la politique migratoire à la réduction du besoin de financement de la protection sociale. Elle estime qu'une politique migratoire ambitieuse contribuerait en 2050 à une réduction du fardeau fiscal lié au vieillissement démographique en 2050 d'un peu plus de $20 \%$ sans critère de sélection et d'environ $30 \%$ avec une politique très sélective (en faveur des travailleurs qualifiés).

24. On doit signaler que le problème du manque de données s'explique par l'absence d'enquêtes ethniques longitudinales en France. En fait, il est exclu de collecter et de traiter des données personnelles faisant apparaître, entre autres, les orientations religieuses et les origines ethniques et raciales, sauf dérogation spéciale exclusivement accordée par la Commission nationale de l'informatique et des libertés (CNIL). 25. Jennifer Hunt, "The impact of the 1962 repatriates from Algeria on the French labor market ", in Industrial \& Labor Relations Review, vol. 45, $n^{\circ}$ 3, 1992, pp. 556-572. 26. Javier Ortega, Gregory Verdugo, " The impact of immigration on the French labor market: Why so different? ", in Labour Economics, vol. 29, 2014, pp. 14-27. 27. Xavier Chojnicki, Lionel Ragot, On entend dire que l'immigration coûte cher à France. Qu'en pensent les économistes?, Paris, Eyrolles, 2012. 